\title{
Dejar de fumar en el trastorno mental grave: desafíos y oportunidades en tiempos de la COVID-19
}

\section{Smoking cessation in severe mental illness: challenges and opportunities in the COVID-19 times}

\author{
Fernando Sarramea*******,****, María José Já́n-Moreno*****,**, \\ ViGENT BaLANZÁ-MARTÍnEZ************. \\ * Instituto Maimónides de Investigación Biomédica de Córdoba (IMIBIC), Córdoba. España. \\ ** Unidad de gestión clínica de Salud Mental. Hospital Universitario Reina Sofía, Córdoba. España. \\ *** Departamento Ciencias Morfológicas y Sociosanitarias, Área de Psiquiatría. Universidad de Córdoba, Córdoba. España. \\ **** Centro de Investigación Biomédica en Red de Salud Mental, Oviedo. España. \\ ***** Unidad docente de Psiquiatría y Psicología Médica, Departamento de Medicina. Universidad de Valencia, Valencia. España. \\ ****** Centro de Investigación Biomédica en Red de Salud Mental (CIBERSAM). \\ Instituto de Salud Carlos III (ISCIII), Madrid. España.
}

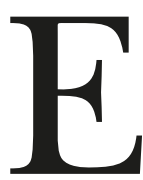

n la actualidad, la enfermedad por coronavirus (COVID-19), propagada rápidamente desde la región china de Wuhan al resto del planeta, amenaza la salud pública mundial. Aunque la mayor parte de los casos se recuperan sin secuelas, en los pacientes de mayor gravedad se asocia con una reacción proinflamatoria que resulta en altas tasas de morbilidad y mortalidad (Yi, Lagniton, Ye, Li y Xu, 2020).

La evidencia disponible sugiere que el tabaquismo y la historia previa de enfermedades metabólicas, cardiovasculares y respiratorias están asociadas, de manera independiente, a un mayor riesgo de insuficiencia pulmonar, ingreso en la UCI y muerte (Wang, Ruobao, Zhong y Huang, 2020; Vardavas y Nikitara, 2020). Por estas razones, la pandemia amenaza con impactar con mayor intensidad a las poblaciones vulnerables como las personas con trastorno mental grave (TMG).

De hecho, las personas con TMG ya tienen un alto riesgo de morbilidad y mortalidad prematuras, lo que reduce su esperanza de vida entre diez y veinte años. En esta población, las enfermedades cardiovasculares y respiratorias son entre dos y tres veces más frecuentes y tienen un inicio más temprano y resultados más graves (De Hert et al.,
2011). El tabaquismo es el principal factor prevenible de riesgo para ambas comorbilidades (Rüther et al., 2014).

El tabaquismo, con mayores niveles de dependencia, en una población con un nivel más elevado de aislamiento social y menor oportunidad de obtener cuidados preventivos, perpetúa este problema todavía más. Son necesarias medidas urgentes tanto sociales como de concienciación médica que puedan facilitar el acceso a tratamientos seguros y efectivos para la cesación tabáquica.

El confinamiento relacionado con la pandemia se ha asociado con unos cambios importantes en el estilo de vida, incluso en los ámbitos de tabaquismo y abuso de sustancias (Balanzá-Martínez, Atienza-Carbonell, Kapczinski y De Boni, 2020; García-Álvarez, de la Fuente-Tomás, Sáiz, García-Portilla y Bobes, 2020). Tras la desescalada progresiva, ahora la prioridad se centra en las medidas preventivas. Esta pueda ser una oportunidad de oro para promover con más ímpetu mensajes relacionados con el cese del tabaquismo. El reto actual implica asegurar que la respuesta a la pandemia y su tratamiento representen una oportunidad para incluir a las personas con un TMG, que tienen mayor vulnerabilidad debido a su posición social y acceso desigual a los servicios sanitarios.

Recibido: Junio 2020; Aceptado: Junio 2020.

Enviar correspondencia a:

María José Jaén-Moreno. Universidad de Córdoba. Área de Psiquiatría. Departamento de Ciencias Morfológicas y Sociosanitarias.

Avda. Menéndez Pidal s/n. Facultad de Medicina y Enfermería. Córdoba, España. Teléfono: +34 957218218.

E-mail: mjjaen@uco.es. 
Aunque los pacientes con un TMG suelen ser menos conscientes de los riesgos para la salud del tabaquismo a largo plazo, hasta el $70 \%$ de estas población ha considerado alguna vez dejar de fumar (Prochaska et al., 2011). Como ocurre con la mayoría de los fumadores, simplemente preguntar al paciente sobre sus hábitos impulsa la motivación para dejarlo. En los enfoques multicomponente, el nivel de motivación alcanzado para la cesación tabáquica es decisivo para reducir el consumo de manera significativa, como paso previo a esta (Sarramea et al., 2019a; Sarramea Crespo et al., 2019b). Es más, información concreta y personalizada sobre los riesgos respiratorios y las probabilidades de prevención podría aumentar la disposición del fumador para dejar de fumar (Sarramea et al., 2019c).

Con la saturación de los servicios sanitarios debido a la pandemia, los profesionales de la salud mental necesitarán adaptarse a los nuevos modelos de atención sanitaria, el aumento de la demanda y las nuevas necesidades de los pacientes. En este contexto, uno de los riesgos principales consiste en limitar de nuevo los enfoques terapéuticos para el TMG a la estabilidad clínica y la prevención de ingresos, por tanto haciendo caso omiso, entre otros, a un objetivo más global de abordar la salud mental y física de los pacientes con un TMG. Se debería tener en cuenta la prevención de los factores ambientales de riesgo, como el tabaquismo, que son determinantes principales de las altas tasas de morbilidad y mortalidad prematura a nivel mundial.

En resumen, la pandemia actual puede ser una oportunidad para valorar tanto la salud como la prevención de enfermedades. Un reto principal es identificar a los grupos más vulnerables y proteger sus necesidades de atención sanitaria a pesar de las emergencias actuales y apremiantes de la COVID-19. Con el fin de prevenir la morbilidad y mortalidad prematuras, es necesario, más que nunca, un enfoque integral y coordinado de la salud mental y física. Esto es de especial importancia para los pacientes con un TMG, que están preparados para recibir un mensaje claro sobre los factores de riesgo del tabaquismo y las soluciones disponibles para ayudarles a dejar de fumar.

\section{Conflicto de interés}

El autor VBM ha sido consultor, asesor o ponente en la educación médica continuada (EMC) durante los últimos tres años para las siguientes empresas: Angelini; Ferrer; Lundbeck; Nutrición Médica; y Otsuka.

Los demás investigadores no tienen intereses biomédicos económicos ni posibles conflictos de interés.

\section{Ayudas económicas}

Los autores no han recibido ninguna ayuda económica para la investigación, autoría o publicación de este manuscrito.

\section{Referencias}

Balanzá-Martínez, V., Atienza-Carbonell, B., Kapczinski, F. y De Boni, R. B. (2020). Lifestyle behaviours during the COVID-19 - time to connect. Acta Psychiatrica Scandinavica, 141, 399-400. doi:org/10.1111/acps.13177.

De Hert, M., Correll, C. U., Bobes, J., Cetkovich-Bakmas, M., Cohen, D., Asai, I., ... Leucht, S. (2011). Physical illness in patients with severe mental disorders. I. Prevalence, impact of medications and disparities in health care. World Psychiatry, 10,52-77. doi:10.1002/j.2051-5545.2011. tb00014.x.

García-Álvarez, L., de la Fuente-Tomás, L., Sáiz, P. A., García-Portilla, M. P. y Bobes, J. (2020). Will changes in alcohol and tobacco use be seen during the covid-19 lockdown?. Adicciones, 32, 85-89. doi:10.20882/adicciones.1546.

Prochaska, J. J., Reyes, R. S., Schroeder, S. A., Daniels, A. S., Doederlein, A. y Bergeson, B. (2011). An online survey of tobacco use, intentions to quit, and cessation strategies among people living with bipolar disorder. Bipolar Disorders, 13, 466-473. doi:10.1111/j.1399-5618.2011.00944.x.

Rüther, T., Bobes, J., De Hert, M., Svensson, T. H., Mann, K., Batra, A., ... Möller, H. J. (2014). EPA guidance on tobacco dependence and strategies for smoking cessation in people with mental illness. European Psychiatry, 29, 65-82. doi:10.1016/j.eurpsy.2013.11.002.

Sarramea, F., Jaen-Moreno, M. J., Balanzá-Martínez, V., Osuna, M. I., Alcalá, J. Á., Montiel, F. J., ... Gutiérrez-Rojas, L. (2019a). Setting the stage to quit smoking in bipolar disorder patients: Brief advice in clinical practice. Adicciones, 31, 136-145. doi:10.20882/adicciones.1006.

Sarramea Crespo, F., Jaén-Moreno, M. J., Gutiérrez-Rojas, L., Balanzá-Martínez, V., García-Alvarez, L., Saiz Martínez, P. A., ... Bobes, J. (2019b). "Readiness to Change" predicts efficacy of reduction among smokers with severe mental illness. European Addiction Research, 25, 256262. doi:10.1159/000500450.

Sarramea, F., Jaén-Moreno, M. J., Feu, N., Redondo-Écija, J., Balanzá-Martínez, V., Gutiérrez-Rojas, L. y García-Portilla, M. P. (2019c). Preparar la cesación tabáquica en el trastorno mental grave: Diagnóstico precoz y oportunidades de prevención. Revista de Psiquiatría y Salud Mental, 12, 133-134. doi:10.1016/j.rpsm.2018.08.001.

Vardavas, C. y Nikitara, K. (2020). COVID-19 and smoking: A systematic review of the evidence. Tobaco Induced Diseases, 18, 1-4. doi:10.18332/tid/119324.

Wang, B., Ruobao, L., Zhong, L. y Huang, Y. (2020). Does comorbidity increase the risk of patients with COVID-19: Evidence from meta-analysis. Aging, 12, 6049-6057. doi:10.18632/AGING.103000.

Yi, Y., Lagniton, P. N. P., Ye, S., Li, E. y Xu, R. H. (2020). COVID-19: What has been learned and to be learned about the novel coronavirus disease. International Journal of Biological Sciences, 16, 1753-1766. doi:10.7150/ijbs.45134. 